\title{
Studying Variation in Cell Apoptosis Caused by Corticosteroids in Thymus of the Rat
}

\author{
Yusef Dustar ${ }^{1}$, Mehrdad Hashemi², Heidar Malayeri ${ }^{3}$, Zahra Ghazi Jahani', \\ Mehrdad Neshat Gharamaleki ${ }^{1}$, Farhad Shaveisi Zadeh ${ }^{4}$,Ali Asghar Karaminia ${ }^{5}$, \\ Abolfazl Movafagh ${ }^{4}$
}

${ }^{1}$ Department of Pathology, The Islamic Azad University, Tabriz Branch, Tabriz, Iran. ${ }^{2}$ Department of Molecular Genetics, Tehran Medical Branch, Islamic Azad University, Tehran, Iran. ${ }^{3}$ The Islamic Azad University, Tabriz Veterinary Branch, Tabriz, Iran. ${ }^{4}$ Department of Medical Genetics, Cancer, Research Center, Shohada Hospital, School of Medicine, Shahid Beheshti University of Medical Science, Tehran, Iran. ${ }^{5}$ Cancer Research Center, Shohada Hospital, School of Medicine, Shahid Beheshti University of Medical Science, Tehran, Iran.

\begin{abstract}
Background and purpose: Apoptosis or planned death of cells is the main mechanism in completion and hemostasis of mature tissues to terminate useless, contaminated, and mutated/damaged cells through internal suicide pathways. One of the agents that creates apoptosis signals is glucocorticoids. As the representative of corticosteroid drugs, dexamethasone can induce apoptosis via internal endonuclease. Therefore, dexamethasone was used as a synthetic glucocorticoid in rats. The effects of dexamethasone on thymocyte of rats, morphological features of apoptotic cells, and the relationship between drug dosage and severity of apoptosis were examined. Methodology: A treatment group with four subgroups (each with five rats) namely T-a, T-b- T-c, and T-a was formed and received IP dexamethasone of $0.5,1.5,2.5,3.5 \mathrm{mg}$ per one-kilogram body weight respectively. In addition, a control group with similar subgroups was formed. Thymus gland of the subjects of both groups was removed six hours after injection. The samples were examined using electron and optical microscopes. Findings: Examinations by optical microscope revealed round or elliptic apoptotic cytoplasmic bodies with or without nucleus basophil materials along with crescentic bodies of chromatin on the apoptotic cells. In addition, electronic microscope images reveled marginal augmentation of nucleus chromatin in osmiophilic forms detached from nucleus fibrillary centers along with a disordered cellular region, endoplasmic reticulum complication, and nucleus fragmentation. There was a direct and significant relationship between the dosage of the drug and the severity of apoptosis. Conclusion and Recommendations: The results showed that standard and higher dosages of corticosteroid stimulated DNA fragmentation in thymocytes and apoptosis of thymocytes induced by corticosteroid is a calcium-depended process.
\end{abstract}

Keywords: Apoptosis- thymus- corticosteroid

Asian Pac J Cancer Biol, 1 (4), 83-87

\section{Introduction}

Apoptosis was first found in genetic studies on nematode Caenorhabditis elegans. Programmed apoptosis throughout the life cycle of the worm included the activity of the selected death gene that exactly kills 131 cells and leaves 959 cell intact. Ker et al. (1972) adopted the term apoptosis from Greek, which literally
Submission Date: 08/13/2016 Acceptance Date: 11/25/2016

means leaves falling down [1-3]. Apoptosis is a sort of programmed cell death and it has recently drawn a great deal of attention of researchers. The reason for this increase of attention on apoptosis is beyond the fact that apoptosis is a key biological phenomenon information and development of tissues and hemostasis. The close relationship between this cellular event and variety of diseases makes apoptosis one of the main research topics.

Corresponding Author:

Dr. Abolfazl Movafagh

Department of Medical Genetics, Cancer Research Center, Shoha Hospital, School of Medicine, Shahid Beheshti University of Medical

Science, Tehran, Iran.

Email: movafagh.a@sbmu.ac.ir 
Cancers and some of the viral diseases, in particular, are of the main concerns in this regard. Uncontrolled cells suicide is to blame as the pathogenesis of a wide range of diseases such as myocardial ischemia and infraction, nervous degenerative changes, Alzheimer's disease, autoimmune disease, and viral infection; in addition, it is highly important in analyzing cancerous tumors. There are four main systems in the initiation of cell apoptosis: 1- signal pathways that initiate a cascade activation of an enzyme called caspases. Signals pathways are formed by connecting dedicated ligands to cell membrane receivers. The receivers are featured with death recognition domains that react with similar or homologs domains named adaptor proteins. The proteins transfer the received signals to the initiation point of apoptosis paths. 2- Cell damage pathway: cell damages initiate apoptosis: increase of penetrability of mitochondria membrane following cell damage and activation of caspases enzymes, the formation of free radicals, cell anoxia, the increase of free calcium level inside the cell, and playing an intermediate role in apoptosis by $\mathrm{T}$ cytotoxic lymphocyte with Perforin/ GranzymeB function. 3- DNA Damage/P53-P73: DNA damage results in augmentation of P53 protein and facilitation of DAN repair by the protein. Should this internal cell pathway fails, P53 protein modifies and initiates transcription of apoptosis supporting factors. 4Cell membrane damage pathway activates sphingomyelin enzyme and generation of ceramide agent out of lipid compounds of the cell membrane. Afterward, the ceramide factor generates apoptosis signal [1-4].

\section{Role of corticosteroids in inducing apoptosis of thymocytes}

Glucocorticoids may induce apoptosis; the level of the agent in thymus tissue is very high, thereby the cells that remain alive in the thymus are featured with signals to stop apoptosis [5-6]. In general, glucocorticoids tend to bind with the receptors called glucocorticoids receptor (GR) in cytoplasm thymocytes and enter the cell nucleus by forming GR complex [7]. Inside the nucleus, the complex reacts with DNA of the cell and increases expression of the Bax gene and generation of Bax protein. The protein moves toward mitochondria and forms specific pore in mitochondria membrane. The pore allows cytochrome $\mathrm{C}$ (apoptotic activating factor-2), which is a key molecule in cell respiration, to dispatch from mitochondria and enter cytoplasm and participate in apoptosis induction pathway. So that it activates an enzyme cascade pathway known as caspases enzymes by cooperating with other molecules such as Apoptotic activating factor-1 and apoptotic activating factor -3 ; the enzymes, in turn, activate endogenous endonuclease enzyme that participates in damaging DNA of the cell $[8,9]$. On the other hand, caspases enzymes split an enzyme called poly ADP Ribose Polymerase (PARP) that normally plays a key role in cell repair; however, by splitting PARP, DNA repair is halted and endonuclease enzyme finds a chance to induce its effects [10-13]. In presence of Bax protein and development of pores in the membrane of mitochondria, an agent called apoptosis-inducing factor (AIF) comes out of mitochondria and induces severe augmentation of chromatin and fragmentation of cell DNA. In addition, by affecting translocase enzyme, AIF deactivates the enzyme and forms phosphatidylserine molecules on the membrane of apoptotic cells by activating Floppase and Scramblase enzymes. Having these molecules formed, apoptotic objects become detectable by macrophage cells [14-18].

\section{Marerials and Methods}

Forty male Wister rats (average weight $=250 \mathrm{gr}$ ) supplied from the department of lab animals, Faculty of Veterinary, The Islamic Azad University-Tabriz were used in the experiments. The rats were grouped into two groups of control and experiment groups $(n=20)$ and each group contained four subgroups $(n=5)$. All the rats were kept in identical condition with minimum stressors (to avoid thymolytic).

As a corticosteroid drug, the treatment group received dexamethasone and the control group receives physiologic serum as a neutral agent. Dexamethasone was supplied from the pharmacy in the form of the $8 \mathrm{mg} / 2 \mathrm{cc}$ human ampoule and stored as recommended by the manufacturer. The drug was diluted by $6 \mathrm{cc}$ distilled water before injection $(0.01 \mathrm{mg}$ drug per unit). The injection was done using the insulin syringe. The subjects were restrained so that the abdominal part of their body was accessible for IP injection. The treatment groups received dexamethasone and the control group received saline serum. The treatment subgroups $\mathrm{T}-\mathrm{a}, \mathrm{T}-\mathrm{b}, \mathrm{T}-\mathrm{c}$, and $\mathrm{T}-\mathrm{d}$ received $0.5,1.5,2.5$, and $3.5 \mathrm{mg} / \mathrm{kg}$ (body weight) respectively. The control subgroups received the equal volume of saline serum.

To obtain specimens of the thymus of the subjects and conduct examination by optical and electron microscopes, the specimen was removed six hours after the injection. [19-20].

\section{Results}

Examination of the treatment samples by optical microscope revealed pathological changes caused by dexamethasone on thymocytes. Crookedness and general condensation were observed in the cells so that the cells were shrunken by $50 \%$ and there were gaps among the cells. The cells demonstrated intense concentration of chromatin along with fragmentation of the nucleus. This indicates apoptosis in thymocytes of the rats' Photomicrographs supported these findings. Nucleus fragmentation and chromatin condensation were clearly evident in the EM samples. There were bubbles on the cell surface as it was boiling. However, one of the most notable changes in the cell was the formation of crescentic chromatin bodies, which is a definite morphological feature of apoptotic cells. The control thymus samples had the normal morphological appearance. The number of apoptotic cells in treatment and control samples in five fields of the optical microscope (40x) and average cell counts were also obtained (Table 1).

As noted earlier, one of the objectives of the study was to examine the relationship between the dosage and 


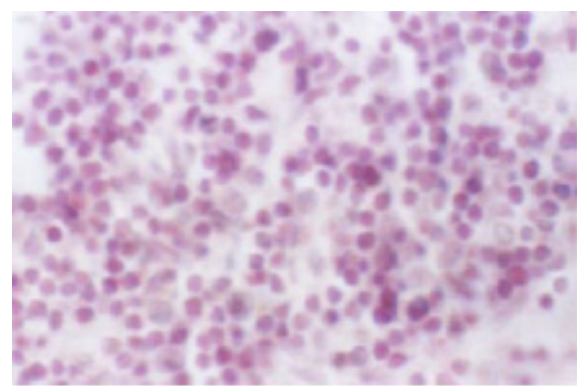

Figure 1. Photomicrograph of the Rat's Thymus Cross Section in the Control Group (H\&E-100x)

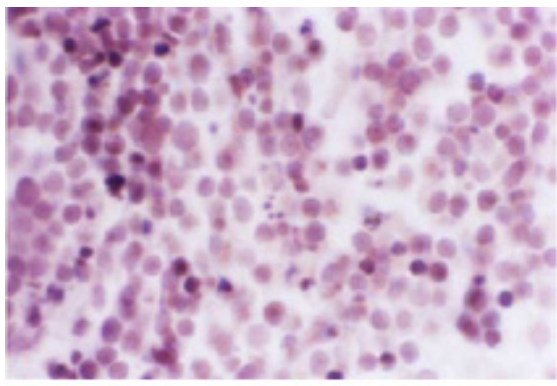

Figure 2. Photomicrograph of the Rat's Thymus Cross Section in Treatment Group after Dexamethasone Injection. Lymphocytes are Denser and Fragmentation of Chromatin is Increased Comparing with the Control Group (H and E-100x)

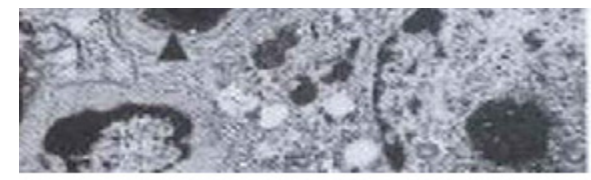

Figure 3. Individual Ultra Photomicrograph of Lymphocyte Cells in the Control Group. Chromatin is Uniform, the Nucleus is Normal in Size and Represents the Largest Portion of Cell's Volume while Cytoplasm is Limited to Peripheral Areas of the Nucleus (Uranyl Acetate- 13600x)

intensity of apoptosis. The number of apoptotic cells in five microscopic fields (Figures 1-5) (40X) were counted and the average number of cells was calculated. Parametric statistic tests (ANOVA) showed a significant relationship between dosage and apoptotic cells count $(p<0.005)$. So that increase in dosage of the medicine increased the number of apoptotic cells.
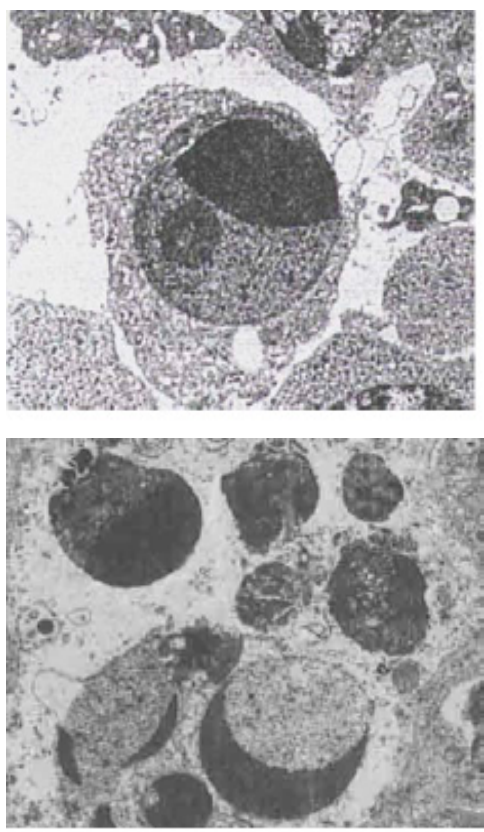

Figure 4. Individual Ultra-Photomicrograph of Thymocyte Cell in Dexamethasone Group; Note Crescentic Forms and Fragmentation of Chromatin of Nucleus (Uranyl Acetate Marking- 12800x)

\section{Discussion}

Weily et al. (1980) reported apoptosis morphological changes of thymocytes in contact with glucocortychostroides. Their best guess was that the steroids stimulated synthesis of one endonuclease [3-15-17].

Corticosteroid agents like dexamethasone stimulated DNA fragmentation in thymocyte of the rat and this happens when the dosage is 2 or 3 times more than standard dosage and apoptosis changes increases due to formation or synthesis of considerable amounts of endogenous endonuclease enzyme. According to studies, glicocortychoides play a key role in keeping the thymus in its natural size so that removing adrenal grand led to the enlargement of the thymus. Therefore, it is clear that glicocortychoides agents are effective on inducing apoptosis of thymus cells [9].

Using different dosages of dexamethasone, probably, causes changes in the distribution of magnesium and calcium inside the cell. The drug activates calcium

Table 1. Average Count of Apoptotic Cells in the Samples

\begin{tabular}{lccc}
\hline Group & Subgroup & Dosage & Average count of apoptotic cells in five microscopic fields (40X) \\
\hline Treatment (dexamethasone) & T-a & 0.5 & 9 \\
& T-b & 1.5 & 9 \\
& T-c & 2.5 & 14 \\
& T-d & 3.5 & 21 \\
Control (saline serum) & S-a & 0.5 & 2 \\
& S-b & 1.5 & 1 \\
& S-c & 2.5 & 3 \\
\hline
\end{tabular}


and magnesium transfer mechanisms and facilitates the transfer of the element to the cell, which results in the activation of endogenous endonuclease enzyme [20-26].

The results showed a significant direct relationship between the intensity of apoptosis changes in thymocytes and dosage of dexamethasone. To explain, thymocyte of the rate has a special steroid receptor that increases expression of apoptosis-inducing gene along with the increase of dosage of the drug [13-14-23]. In general, dexamethasone induces its effect through attaching to GRs, which are bound with thermal shock proteins that prevent conformational changes in gulicocorticostroide. However, in presence of standard or high dosage of dexamethasone inside thymus cell that attaches to GRs, conformational changes are triggered, a homodimer is formed by dexamethasone, and GR and the drug find its way inside the nucleus of the cell. This results in an increase of expression of Bax gene and formation of permeability transition pore complex (PTPC). Due to the presence of the gene at mitochondria membrane, a large volume of cytochrome $\mathrm{C}$ and AIF is discharged from mitochondria and caspases enzymes are activated [2], [8-10]. Eventually, thymocyte apoptosis is triggered by different dosages of the drug.

In short, different dosages of dexamethasone is effective on apoptosis changes as it increases distribution of calcium and magnesium on one hand and increases expression of apoptosis-inducing genes on the other hand, which result in an increase in the intensity of apoptosis changes caused by dexamethasone [7-9].

\section{Acknowledgments}

This research work is part of the thesis of Msc student Yusef Dustar. The authors wish to thank Department of Research and Medicine, The Islamic Azad University.

\section{References}

1. Allen RT, Hunter WJ, 3rd, Agrawal DK. Morphological and biochemical characterization and analysis of apoptosis. Journal of pharmacological and toxicological methods. 1997;37(4):215-28.

2. Kerr JF, Wyllie AH, Currie AR. Apoptosis: a basic biological phenomenon with wide-ranging implications in tissue kinetics. British journal of cancer. 1972;26(4):239-57.

3. Wyllie AH, Kerr JF, Currie AR. Cell death: the significance of apoptosis. International review of cytology. 1980;68:251306.

4. Steller H. Mechanisms and genes of cellular suicide. Science (New York, NY). 1995;267(5203):1445-9.

5. Krenger W, Rossi S, Hollander GA. Apoptosis of thymocytes during acute graft-versus-host disease is independent of glucocorticoids. Transplantation. 2000;69(10):2190-3.

6. Lechner O, Wiegers GJ, Oliveira-Dos-Santos AJ, Dietrich H, Recheis H, Waterman M, et al. Glucocorticoid production in the murine thymus. European journal of immunology. 2000;30(2):337-46.

7. Tonomura N, McLaughlin K, Grimm L, Goldsby RA, Osborne BA. Glucocorticoid-induced apoptosis of thymocytes: requirement of proteasome-dependent mitochondrial activity. Journal of immunology (Baltimore, Md : 1950).
2003;170(5):2469-78.

8. Nakamura M, Yagi H, Ishii T, Kayaba S, Soga H, Gotoh $\mathrm{T}$, et al. DNA fragmentation is not the primary event in glucocorticoid-induced thymocyte death in vivo. European journal of immunology. 1997;27(4):999-1004.

9. Tarcic N, Ovadia H, Weiss DW, Weidenfeld J. Restraint stress-induced thymic involution and cell apoptosis are dependent on endogenous glucocorticoids. Journal of neuroimmunology. 1998;82(1):40-6.

10. Dimri R, Sharabi Y, Shoham J. Specific inhibition of glucocorticoid-induced thymocyte apoptosis by substance P. Journal of immunology (Baltimore, Md : 1950). 2000;164(5):2479-86.

11. Gruber J, Sgonc R, Hu YH, Beug H, Wick G. Thymocyte apoptosis induced by elevated endogenous corticosterone levels. European journal of immunology. 1994;24(5):111521.

12. Hegardt C, Andersson G, Oredsson SM. Changes in polyamine metabolism during glucocorticoid-induced programmed cell death in mouse thymus. Cell biology international. 2000;24(12):871-80.

13. Hegardt C, Andersson G, Oredsson SM. Different roles of spermine in glucocorticoid- and Fas-induced apoptosis. Experimental cell research. 2001;266(2):333-41.

14. Hegardt C, Andersson G, Oredsson SM. Spermine prevents cytochrome c release in glucocorticoid-induced apoptosis in mouse thymocytes. Cell biology international. 2003;27(2):115-21.

15. Hirano T HH, Ishishita H, Uda S, Oka K. . Oxidized glucocorticoids counteract glucocorticoid-induced apoptosis in murine thymocytes in vitro. . Life Sci. 2001; ; 68((26): 2905-16.

16. Islam Z, Nagase M, Yoshizawa T, Yamauchi K, Sakato N. T-2 toxin induces thymic apoptosis in vivo in mice. Toxicology and applied pharmacology. 1998;148(2):205-14.

17. Iwata M. [Thymocyte apoptosis and thymic selection]. Seikagaku The Journal of Japanese Biochemical Society. 1994;66(6):521-5.

18. Lutz CT, Browne G, Petzold CR. Methylcholanthrene causes increased thymocyte apoptosis. Toxicology. 1998;128(2):151-67.

19. Chmielewski V, Drupt F, Morfin R. Dexamethasone-induced apoptosis of mouse thymocytes: prevention by native 7alpha-hydroxysteroids. Immunology and cell biology. 2000;78(3):238-46.

20. Cifone MG, Migliorati G, Parroni R, Marchetti C, Millimaggi D, Santoni A, et al. Dexamethasone-induced thymocyte apoptosis: apoptotic signal involves the sequential activation of phosphoinositide-specific phospholipase C, acidic sphingomyelinase, and caspases. Blood. 1999;93(7):228296.

21. Asada A, Zhao Y, Kondo S, Iwata M. Induction of thymocyte apoptosis by $\mathrm{Ca} 2+$-independent protein kinase $\mathrm{C}(\mathrm{nPKC})$ activation and its regulation by calcineurin activation. The Journal of biological chemistry. 1998;273(43):28392-8.

22. Berki T, Palinkas L, Boldizsar F, Nemeth P. Glucocorticoid (GC) sensitivity and GC receptor expression differ in thymocyte subpopulations. International immunology. 2002;14(5):463-9.

23. Dallaporta B, Marchetti P, de Pablo MA, Maisse C, Duc HT, Metivier D, et al. Plasma membrane potential in thymocyte apoptosis. Journal of immunology (Baltimore, Md : 1950). 1999;162(11):6534-42.

24. TD. A. Ultrastructural aspects of cell death. In: Potten CS,. Oxford. ePomcdOUP, editor1987. 39-65. p.

25. Miura N, Yamamoto M, Ueki T, Kitani T, Fukuda K, 
Komatsu Y. Inhibition of thymocyte apoptosis by berberine.

Biochemical pharmacology. 1997;53(9):1315-22.

26. Nagy P PG, Jenei A, Berne L, Gaspar R Jr, Matko J, et al. . Ion-channel activities regulate transmembrane signaling in thymocyte apoptosis and T-cell activation. Immunol Lett 1995;44((2-3):):91-5.

\section{(ब) $\odot \otimes$}

This work is licensed under a Creative Commons AttributionNon Commercial 4.0 International License. 